\title{
REGISTER RERUM ad Vol. 38
}

Confecit G. Boehm, Basel

A-cells, v. Langerhans' islets

Adipeux, tissu adipeux, v. Age changes

Adrenaline, v. Adrenergetic nerves, Catechol amines

Adrenals, v. Nebennierenrinde

Adrenergic nerves, demonstration, with the osmic acid-sodium iodide

technique $\quad 379$

Age changes in fat distribution in the female body 56

$$
\text { ;(C) } 173
$$

- ; v. Knochennaht

Aile (embryon de poulet), v. Wing bud

Alterations des nerfs périphériques après la mort, v. Nerven, periphere

Altern, v. Age changes, Knochennaht

Alveolärer Profil·winkel, Beziehungen zu den vorderen Trajektorien des

Splanchnocraniums (C) 167

Ameisenbär, brasilianischer, v. Paneth cells

Anatomie des Menschen, Lehrbuch (1. Bd.: Allgemeine Anatomie und Be-

wegungsapparat (6. Aufl.); 3. Bd.: Nervensystem, Haut und Sinnes

organe (5. Aufl.) (B) 177

Anatomie, mikroskopische, des Menschen, Handbuch (Bd. 3: Haut und

Sinnesorgane, 3. Teil: Haut; Milchdrüse; Ergänzung zu Bd. III/l) (B) 181,182

Anatomie; les nouveaux termes anatomiques (Lexique conforme à la nomen

clature Internationale PNA) (B) . 184

Anatomistes yougoslaves, reunion annuelle (1958), comptes-rendus (C) . . $160 \mathrm{ff}$.

Anatomy; Strong andElwy'́'s human neuroanatomy (4th ed.) (B) 186

Anatomy for surgeons (Vol. 111: The back and limbs) (B) 385

Anchyotie, v. Schädelentwicklung

Anencephalia, v. Tératogénèse

Anneaux fibreux auriculoventriculaires, contribution à leur structure histo-

logique (C) 164

Ant bear (brazilian), v. Tamandua tetradactyla

Anterior chamber (of the tawny owl), v. Metachromasia

Anthozyan und Rosolsäure in der Zytologie (C) 166

-, v. Zellkern

Antikörper, fluoreszenz-markierte, v. Histochemie

Anuli fibrosi, v. Anneaux fibreux auriculoventriculaires

«Apical ridge», role in the development of the chick embryo limb bud . . . 280

Apparatus digestorius, v. Digestive tract 
Aqueous humor; demonstration of metachromasia and its distribution in the anterior chamber of the tawny owl (Síri $\lambda$ : aluco) using a new printing technique on plaster slides $\quad 140$

Arachnoidea, v. Cisterna ponto-cerebellaris

Area striata, v. Sehrinde

Arterial supply of the bladder and ureter following radical hysteroectomies (C) 172

Arterio-venöse Anastomosen; Anatomie, Biologie, Pathologie (2. Aufl.) (B) 180

Arthritis, v. Spinal joint changes

Arthrosis deformans, v. Spinal joint changes

Articulatio genu, v. Articulation du genou, Tibia

$1(\mathrm{~B})=$ Book reviews - Livres nouveaux - Buchbesprechungen $(\mathrm{C})=$ Report - Communication

- Vortrag

390

Register rerum

Articulation dugenou; morphologie et function des ligaments ménisco-fémo-

raux de 1'articulation du genou chez Thome 304

Articulationes intervertebrales, v. Spinal joint changes

Aseptic conditions, v. Wounds

Atlas, v. Röntgenbild, Zytodiagnostik

Auge, v. Malformations, Metachromasia

Auris interna, v. Bogengang

Autonome Nerven, v. Adrenergic nerves, Digestive tract

Autoradiographie, v. Histochemie, Spleen

Aves, v. Taubenleber, Wing bud

Back, v. Surgeons

Bauchspeicheldrüse, neue Befunde zu ihrer Entwicklung 114

Beaver, v. Biber

Bewegungsapparat, v. Anatomie

Biber; Anatomie des Hypothalamus des Bibers (Castor canadensis), nebst

morphologischen Aspekten der Neurosekretion 126

Bile ducts; the distribution pattern of the small intrahepatic human bile ducts 63

Biochemisches Taschenbuch (B) 184

Biological microtechnique, principles (A study of fixation and dyeing) (B) . 178

Biological ultrastructure (B) 180

-, v. Zähne, Zahngewebe

Biologische Resorptionen, v. Resorptionen

Biopsy, v. Muscle

Birefringence, v. Dentes, Nebennierenrinde, Zahngewebe

Bladder, vascularisation, in connection with haematuria vesicalis (C) ... 160

-, v. Arterial supply

Blastogenese des Menschen (B) 288

Blessures (des muscles), v. Wounds Blood, v. Blutgefäße, Spleen, Vessels

Blutgefäße, fetale, Polykaryozyten (C)

163

Body (fat distribution), v. Age changes

Bæuf, v. Bovis

Bogengang, hinterer, beim Menschen, Beitrag zur Kenntnis seiner Lage (C) . 165 
Bones, v. Knochen, Knochennaht, Os, Polykaryozyten, Tibia

Bovine embryos, v. Ureteranomalien

Bovis, v. Haematuria vesicalis, Hypophysis, Ureteranomalien

Brain; on the cellular localization of catechol amines (noradrenaline and dopamine) in the brain 277

-; v. Malformations

Canal de Hunter, v. Canalis adductorius

Canalis adductorius; contribution à $\Gamma$ étude morphologique et topographique

du canal de Hunter (C) 168

Canalis semicircularis posterior, v. Bogengang

Canis famíliaris; données histochimiques sur les cellules parafolliculaires de

la glande thyroïde du chien $\quad 332$

-; v. Gefäßarchitektur

Canaux biliaires intrahépatiques, v. Bile ducts Camera bulbi anterior, v. Metachromasia

Carcinom, v. Krebsforschung

Register rerum391

Cardiac necroses, chemical prevention (B) 289

Carence en oxygène, $v$. Tératogénèse

Caries, v. Zahngewebe

Castor, v. Hypothalamus

Castor canadensís, v. Hypothalamus

Cat; development of the deciduous cheek teeth of the cat 187

Cat; postnatal changes in volumetric and density relationships of neurons

in cerebral cortex of cat 291

Catechol amines, (noradrenaline and dopanrine), cellular localization in the brain 277

Cattle, horned cattle, v. Boris

Cavia porcellus, v. Nebennierenrinde, Spleen

Cavum nasi, v. Cornet moyen

Cavum peritonaei hepatis dorsale sinistrum, v. Taubenleber

Cebocephalie, v. Schädelentwicklung

Cell metabolism, regulation, CIBA-Foundation symposium (B)

Cellules du cornet moyen, v. Cornet moyen

Cementum, v. Dentes

Centre visuel, v. Sehrinde

Cephalothoracopagus (rabbit), v. Schädelentwicklung

Cerebellum; les prolongements antérieurs de la citerne ponto-cérébelleuse (C) 173

-; la faux du cervelet, sa morphologie et son contenu (C) 162

Cerebrum; on the cellular localization of catechol amines (noradrenaline and

dopamine) on the brain 277

-; malformations encéphaliques et microphtalmie gauche après section des

vaisseaux vitellins gauches chez Tembryon de poulet

-; postnatal changes in volumetric and density relationships of neurons in cerebral cortex of cat 291

-; topométrie cranio-encéphalique chez Thomme (B)

185

-; v. Cerebellum, Chlorpromazine, Sehrinde, Hypothalamus, Malformations

Cervelet, v. Cerebellum 
Chambre antérieure (chez le chat-huant), v. Metachromasia

Champy's technique (osmic acid-sodium iodide technique), v. Adrenergic nerves

Chat, v. Cat

Chat-huant, v. Aqueous humor

Cheek teeth, deciduous, of the cat, development $\quad 187$

Chemical prevention of cardiac necroses (B) 289

Chick embryo; on the role of the «apical ridge» in the development of the

chick embryo limb bud 280

; v. Malformations, Tératogénèse

Chien; données histochimiques sur les cellules parafolliculaires de la glande

thyroïde du chien 332

-, v. Gefäßarchitektur Chirurgie, v. Anatomy Chitinase, v. Paneth cells

Chlorpromazine, effet sur le noyau supraoptique (C). . . 167

Chromaffine reaction, v. Catechol amines

Chromatin; Sex-Chromatin-Forschung (C) 175

CIBA-Foundation symposium on the regulation of cell metabolism (B) . . 185

Cisterna ponto-cerebellaris; les prolongements antérieurs de la citerne ponto-

cérébelleuse $(\mathrm{C}) \quad .173$

Cobaye, v. Cavia porcellus

392

Register rerum

Cæur, v. Cardiac necroses, Cor

Colonne vertébrale, articulations, v. Spinal joint changes

Coloration, v. Adrenergic nerves, Anthozyan, Langßrhans, islets, Microtechnique

Columba, v. Taubenleber

Columna vertebralis, v. Intervertebral discs, Spinal joint changes

Comptes-rendus de la reunion annuelle des anatomistes yougoslaves (1958) (C) $160 \mathrm{fF}$.

Concha nasalis media, v. Cornet moyen

Condylus lateralis tibiae, Furchen, und Sehnenimpression des lateralen

Meniscus (C) 164

Congélation-dessication, v. Histochemie

Cor; contribution à l'étude de la structure histologique des anneaux fibreux

auriculoventriculaires (C) 164

$-;$ v. Cardiac necroses

Cornet moyen, pneumatisation (morphologie, topographie, intérêt pratique)

(C) 170

Corps gras (tissu adipeux), v. Age changes

Corps vitré, v. Metachromasia

Corpus luteum graviditatis in hare, a contribution to the knowledge of

histology (C) 161

Corpus luteum, v. Zander

Corpus pineale, v. Epiphysis cerebri

Corpus vitreum, v. Metachromasia

Cortex cerebri; postnatal changes in volumetric and density relationships of neurons in cerebral cortex of cat 291

$-;$ v. Sehrinde 
Corticosteroides, v. Cardiac necroses

Cranium; topométrie cranio-encéphalique chezl'homme (B) 185

Cranium, v. Schädelentwicklung, Sinus lateral, Splanchnocranium

«Crete épidermique apicale», v. «Apical ridge»

Cutis, v. Haut

Cycle sexuel, v. Ganglion ovarien

Cytochemical methods, general (Vol. 1) (B) 388

-; v. Histochemie, Handbuch

Cytochemistry, v. Adrenergic nerves, Catechol amines, Corpus luteum, Histochemie,

Histochimie, Paneth cells, Wounds

Cytoplasmic-nuclear ratio, v. Neurons

Cyto..., v. Zyto...

Deciduous cheek teeth of the cat, development

Degeneration, v. Nervous elements

Densité des cellules, v. Neurons

Density and volumetric relationships, v. Neurons

Deformities, v. Malformations, Radicula, Schädelentwicklung, Tératogénèse

Dental focal infections, v. Dental infections

Dental infections and spinal joint changes, Supplementum 36 (=1 ad Vol. 38)

Denies; polarisationsoptische Untersuchung des Zementes entkalkter, retinierter Zähne $\quad 368$

-; v. Dental infections, Radicula endomolaris, Zahngewebe

Dentes decidui; development of the deciduous cheek teeth of the cat .... 187

Dents humain enclusés, v. Dentes

Register rerum

393

Dents de lait, v. Dentes decidui

Development of the deciduous cheek teeth of the cat .

187

Development of the chick embryo limb bud, role of the « apical ridge » ... 280

Development of Radicula endomolaris (C) 170

Development, v. Ontogenesis, Ontogenetic development

Diagnosis, v. Gynäkologische Zytologie, Zytodiagnostik

Difformitées, v. Malformations, Radicula, Schädelentwicklung, Tératogénèse

Digestive tract; contribution to the study of degenerative and regenerative

changes in the intrinsic nervous elements of the digestive tract under

experimental conditions (C) 163

Disci intervertebrales, v. Nerve terminations, Spinal joint changes

Dog, v. Canis familiaris

Dopamine, v. Catechol amines

Doppelbrechende Substanzen der Nebennierenrinde bei experimentell ausgelöstem epileptischem Anfall (Nebennierenrinde und Elektroschock I.) 353

Doppelbrechung, v. Dentes, Zahngewebe

Dorsum, v. Surgeons

Dos, v. Surgeons

Dottersackgefäße, v. Malformations

Double refraction, v. Dentes, Nebennierenrinde, Zahngewebe 
Ductuli biliferi, v. Bile ducts

Ductus semicircularis posterior, v. Bogengang

Dünndarm, v. Paneth cells

Dyeing, v. Staining

Ear, v. Bogengang

Ecorce cérébrale, v. Cortex cerebri

Edentata, v. Tamandua tetradactyla

Eiweißstrukturen des Zellkerns, ihre morphologische Erfassung mittels

Anthozyan-Rosolsäure (C) 166

Elastometrie der Haut beim Menschen (C) 169

Elektroschock und Nebennierenrinde (I. Das Verhalten der doppelbrechen-

den Substanzen der Nebennierenrinde bei experimentell ausgelöstem

epileptischem Anfall) 353

Embryo, v. Bauchspeicheldrüse Chick embryo, Dentes decidui, Malformations, Polykaryozyten, Tératogénèse, Ureteranomalien

Encéphalique, v. Topométrie

Encéphaliques; malformations encéphaliques et microphtalmie gauche après

section des vaisseaux vitellins gauche chez Tembryon de poulet ... 1

Encephalon, v. Cerebrum

Entwicklung, v. Ontogenesis

Eosin; a demonstration of the specific affinity of eosin to the A-cells of the

Langerhans, islets (C) 171

Epileptic attacks, experimentally induced, v. Elektroschock

Epileptischer Anfall, v. Elektroschock

Epiphysis cerebri; effet de $\Gamma$ extrait epiphysaire sur la structure hypothala-

mique (C) $\quad 167$

«Epitheleinschlüsse», v. Lymphknoten

Epithelium; studies on the morphology and histochemistry of the Fallopian tube epithelium,

Supplementum 37 (=2 ad Vol. 38)

Euthermic rat, v. Thyreoidea

394

Register rerum

Extremitates, v. Surgeons, Tibia, Wing bud Eye, v. Malformations, Metachromasie

Färbung, v. Adrenergic nerves, Anthozyan, Langerhans, islets, Microtechnique

Fallopian tube; studies on the morphology and histochemistry of the Fallopian tube epithelium,

Supplementum 37 (=2 ad Vol. 38)

Falx cerebelli, v. Faux du cervelet

Fat distribution in the female body, age changes $\quad 56$

;(C) 173

Faux du cervelet, sa morphologie et son contenu (C) 162

Feinstruktur, v. Ultrastructure, Zähne, Zahngewebe

Fel, v. Bile ducts

Felís domestíca, v. Cat

Feldhase, v. Corpus luteum

Female body, age changes in fat distribution 56

;(C) 173 
Femme, v. Female body, Lymphknoten

Femur, v. Articulation du genou

Ferments, v. 5-Nucleotidase, Paneth cells

Fertility, v. Tuba uterina

Fetale Blutgefäße, v. Blutgefäße

Fettgewebe, v. Age changes

Fibrin; die Rolle des Fibrins bei der Bildung der menschlichen Placenta . . 323

Fissura intraoccipitalis posterior, v. Knochennaht

Fixation, v. Microtechnique

Flügelanlage, v. Wing bud

Fluoreszenz-markierte Antikörper, v. Histochemie

Fluoreszenzmikroskopie, v. Histochemie

Foci; dental focal infections, v. Dental infections

Foie, v. Bile ducts, Taubenleber

Fonction, v. Ligaments ménisco-fémoraux

Fourmillier, v. Paneth cells

Frau, v. Female body, Lymphknoten

Freezing-drying, v. Histochemie

Funktion, v. Ligaments ménisco-fémoraux

Gallenwege, intrahepatische, v. Bile ducts

Gallus gallus, v. Chick embryo, Ganglion ovarien, Malformations, Térato-génèse

Ganglion ovarien de poule au cours du cycle sexuel (C) $\quad 168$

Gefäßarchitektur der Mere. Untersuchungen an der Hundeniere (Heft 5 der

«Zwanglosen Abhandlungen aus dem Gebiet der normalen und patho-

logischenAnatomie»)(B) 387

Gefäße, v. Arterial supply, Arterio-venöse Anastomosen, Blutgefäße, Malformations, Sinus

lateral, Vascularisation

Gefäße (Dottersackgefäße), v. Malformations

Geflügelleber, v. Taubenleber

Gefriertrocknung, v. Histochemie

Gehirnmißbildungen, v. Malformations

Gelbkörper des Zanders Lucíoperca sandra (C) $\quad 174$

Register rerum

395

Genou, v. Articulation du genou, Tibia

Gerichtliche Medizin, v. Knochennaht

Gesellschaftsberichte, v. Yougoslavie

Gips-Objektträger, v. Metachromasia

Glande mammaire, v. Mikroskopische Anatomie

Glandula mammaria, v. Mikroskopische Anatomie

Glandula thyreoidea, v. Thyreoidea

Glandulae intestinales, v. Paneth cells

Glaskörper, v. Metachromasia

Gliedmaßen, v. Surgeons

Graisse (tissu adipeux), v. Age changes

Gravidität, v. Corpus luteum 
Guinea-pig, v. Cavía porcellus

Gynäkologische Zytodiagnostik, Atlas und Leitfaden (B) 386

Gynäkologische Zytologie (Beiträge zur Krebsforschung Bd. 4) (B) .... 183

Haematuria vesicalis (bovis); vascularisation of bladder in connection with haematuria vesicalis $(\mathrm{C}) \quad 160$

Härte, v. Haut

Handbuch der Histochemie (Bd. I: Allgemeine Methodik, 1. Teil: Gefrier-

trocknung, Fluoreszenzmikroskopie, Polarisationsmikroskopie, Mikro-

spektrophotometrie, Mikroradiographie, Autoradiographie, Immun ·

histologie) (B) 387

-; v. Cytochemical methods

Handbuch der mikroskopischen Anatomie des Menschen (Bd. 3: Haut und

Sinnesorgane, 3. Teil: Haut; Milchdrüse; Ergänzung zu Bd. III/I) (B), 181, 182

Hare, v. Corpus luteum

Harnblase, v. Bladder, Hysteroectomies

Haut; die durchschnittliche Dicke der menschlichen Haut (C)

-; Haut des Menschen, Beitrag zu deren Elastometrie (C) 169

-; v. Anatomie, Mikroskopische Anatomie

Heart, v. Cardiac necroses, Cor

Hen, v. Gallus gallus

Hepar; the distribution pattern of the small intrahepatic human bile ducts . 63

-; v. Taubenleber

Hernie inguinale; le facteur osseux dans Гétiologie de la hernie inguinale (C) 170

Herz, v. Cardiac necroses, Cor

Hiatus tendineus, v. Canalis adductorius

Histochemical demonstration of adrenergic nerves with the osmic acid-sodium

iodide technique 379

Histochemie, Handbuch (Bd. I: Allgemeine Methodik; 1. Teil: Gefrier-

trocknung, Fluoreszenzmikroskopie, Polarisationsmikroskopie, Mikro-

spektrophotometrie, Mikroradiographie, Autoradiographie, Immun-

histologie) (B) 387

-; v. Cytochemical methods

Histochemistry; studies on the morphology and histochemistry of the Fallopian tube epithelium,

Supplementum 37 (=2 ad Vol. 38)

-; v. Adrenergic nerves, Catechol amines, Corpus luteum, Cytochemical methods, Histochemie,

Histochimie, Paneth cells, Wounds

Histochimie; données histochimiques sur les cellules parafolliculaires de la

glande thyroïde du chien 332

396

Register rerum

Histologie des Menschen, Leitfaden (2. Aufl.) (B) 289

Histologie, v. Microtechnique, Mikroskopische Anatomie Hormones, v. Catechol amines,

Elektroschock, Epiphysis, Wounds Hühnerembryo, v. Chick embryo, Malformations,

Tératogénèse Huhn, v. Gallus gallus

Human neuroanatomy (Strong and Elwyn) (4th ed.) (B) 186

Humeur vitrée, v. Metachromasia 
Humor aqueus, v. Metachromasia

Hund, v. Canís familiaris

Hundeniere, v. Gefäßarchitektur

Hunter; canal de Hunter, v. Canalis adductorius

Hyaluronic acid, v. Aqueous humor

Hypophysis; der mikroskopische Aufbau der Pars tuberalis der Rinder-

Hypophyse (C) 161

-; v. Hypothalamus

Hypothalamus des Bibers (Castor canadensis), Anatomie, nebst morphologi-

schen Aspekten der Neurosekretion 126

Hypothalamus; contribution à I'étude de I'influence du son sur la structure

de Thypothalamus du rat (C) 174

-; effet de l'extrait epiphysaire sur la structure hypothalamique (C) .... 167

-; v. Catechol amines

Hysteroectomies; arterial supply of the bladder and ureter following radical

hysteroectomies (C) 172

ïmmunhistologie, v. Histochemie Induktion, v. Wing bud Infections, v. Dental infections

Innervation of muscle. A biopsy study (B) 179

Integumentum, v. Anatomie, Haut

Intervertebral discs of man, nerve terminations, ontogenetic development

(Histology of intervertebral discs, 11th communication) 96

-; v. Spinal joint changes Intervertebral discs, v. Spinal joint changes Intestinum tenue, v.

Digestive tract, Paneth cells

Intrahepatic human bile ducts, small, distribution pattern

63

Iodine, radioactive, $v$. Thyreoidea

Isotope, radioaktive, $\mathrm{v}$. Phosphorus, Thyreoidea

J131, v. Thyreoidea

Joints; spinal joint changes and dental infections, Supplementum 36 (=1

ad Vol. 38) Juncturae columnae vertebralis, v. Spinal joint changes

Kaninchen, v. Adrenergic nerves, Catechol amines, Schädelentwicklung Kardiale Nekrosen, v.

Cardiac necroses

Karger, Heinz, Dr. Dr. med. h. c., $\uparrow \quad$ vor 1

Karies, v. Zahngewebe

Karzinom, v. Krebsforschung

Kardiale Nekrosen, v. Cardiac necroses

Karzinom, v. Krebsforschung

Katecholamine (Noradrenalin und Dopamin), v. Brain

Register rerum

397

Katze, v. Cat

Kephalothorakopages Kaninchen, v. Schädelentwicklung

Kern, v. Zellkern

Kern-Zytoplasma Verhältnis, v. Neurons

Kidney, v. Niere, Ureteranomalien

Knee, v. Tibia

Knee joint, v. Articulation du genou, Tibia 
Kniegelenk, v. Articulation du genou, Tibia

Knochen; mikroskopische Untersuchungen der biologischen Resorptionen in den Röhrenknochen (B) 386

-; v. Os, Polykaryozyten, Tibia

Knochennaht; über eine persistierende, für die gerichtlich-medizínische

Bestimmung des Lebensalters bedeutungsvolle Knochennaht (C) . . 171

Körpergewebe (Fettgewebe), v. Age changes

Kompressibilität, v. Haut

Krebsforschung, Beiträge(Bd. 4: Gynäkologische Zytologie) (B) 183

Langerhans' islets; a demonstration of the specific affinity of eosin to the A-

cells of the Langerhans, islets (C) 171

Lapin, v. Adrenergic nerves, Catechol amines, Schädelentwicklung

Leber; Beitrag zur Kenntnis der Morphologie der Geflügel-, insbesondere

Taubenleber (C)

172

-; v. Bile ducts

Lehrbuch der Anatomie des Menschen (1. Bd.: Allgemeine Anatomie und

Bewegungsapparat (6. Aufl.); Bd. 3: Nervensystem, Haut und Sinnes-

organe (5. Aufl.) (B) 177

Lehrbuch, v. Handbuch, Leitfaden, Praxis, Röntgenbild, Surgeons, Zyto-diagnostik

Leitfaden der Histologie des Menschen (2. Aufl.) (B) 289

-, v. Lehrbuch

Leitfaden, v. Zytodiagnostik

Lepus europaeus, v. Corpus luteum

Létal. effet létal, v. Tératogénèse

Lièvre, v. Corpus luteum

Ligamenta menisco-femoralia, v. Ligaments ménisco-fémoraux

Ligaments ménisco-fémoraux de l'articulation du genou chez Thomme,

morphologie et fonction $\quad 304$

Lien, v. Spleen

Limb bud of the chick embryo, development, role of the «apical ridge» . . 280

Limbs, v. Surgeons

Lipoide, v. Doppelbrechende Substanzen

Liver, v. Bile ducts, Taubenleber

Lucioperca sandra, v. Zander

Lymphocytes, v. Spleen

Lymphknoten, pelvine, bei Frauen, glanduläre Einschlüsse (C) 165

Mâchoire supérieur, v. Schädelentwicklung

Malformations encéphaliques et microphtalmie gauche après section des

vaisseaux vitellins gauches chez $\Gamma$ embryon de poulet

1

Malformations, v. Mißbildung, Radicula, Tératogénèse Mamma, v. Mikroskopische Anatomie 398

Register rerum

Mammalia, v. Sehrinde

Mammary gland, v. Mikroskopische Anatomie

Man, v. Age changes, Anatomie, Bauchspeicheldrüse, Bile ducts, Blasto-genese, Bogengang,

Cor, Dentes, Haut, Histologie, Intervertebral discs, Ligaments ménisco-fémoraux, Lymphknoten, 
Mikroskopische Anatomie, Muscle, Neuroanatomy, Placenta, Polykaryozyten, Rönt-genbild, Sehrinde, Spinal joint changes, Topométrie, Tuba uterina, Zahngewebe

Mandibula, v. Splanchnocranium

Maus, v. Mus musculus

Maxilla, v. Schädelentwicklung, Splanchnocranium

Mediaeval skeletons, v. Spinal joint changes

Meerschweinchen, v. Cavia porcellus

Membra, v. Surgeons

Membres, v. Surgeons

Meniscus lateralis (medialis), v. Ligaments ménisco-fémoraux

Meniscus lateralis, v. Tibia

Mensch;Blastogenese(B) 288

-; Röntgenbild des normalen Menschen (Atlas und Lehrbuch für Praxis

und Klinik) (B) 179

Metabolism; CIBA-Foundation symposium on the regulation of cell meta

bolism (B) 185

-; v. Thyreoidea

Metachromasia; demonstration of metachromasia and its distribution in the anterior chamber of the tawny owl (Strix aluco) using a new printing technique on plaster slides 140

Methode, v. Adrenergic nerves, Anthozyan, Aqueuos humor, Cytochemical methods,

Histochemie, Microtechnique, Mikrophotographie, Zyto-diagnostik

Microphtalmie, v. Malformations

Microtechnique, biological, principles (A study of fixation and dyeing) (B) 178

Micro..., v. Mikro...

Mikrophotographie, Praxis (2. Aufl.) (B) 385

Mikroradiographie, v. Histochemie

Mikroskopische Anatomie des Menschen, Handbuch (Bd. 3: Haut und

Sinnesorgane, 3. Teil: Haut; Milchdrüse; Ergänzung zu Bd. III/l) (B) 181,182

Mikroskopische Anatomie, v. Histologie

Mikrospektrophotometrie, v. Histochemie

Mikro..., v. Micro...

Milchdrüse, v. Mikroskopische Anatomie

Milchzähne, v. Dentes decidui

Milk-teeth, v. Dentes decidui

Milz, v. Spleen

Mißbildung; ein Beitrag zur Schädelentwicklung und zum Symmetriepro-

blem auf Grund von Untersuchungen am Schädel eines anchyoten,

prosophthalmen, kephalothorakopagen Kaninchens mit rudimentärer

Oberkieferanlage 213

-; v. Malformations, Radicula, Tératogénèse

Molares, v. Dentes decidui

Monosymmetrie, v. Schädelentwicklung

Monstre céphalothoracopage, v. Schädelentwicklung

Register rerum399

Morphogenese, v. Schädelentwicklung 
Motor cortex, v. Cortex cerebri

Mouse, v. Mus musculus

Mus musculus; contribution à Tétude de Faction du son sur le testicule de la souris (C) $\quad 176$

Muscle; the innervation of muscle. A biopsy study (B) 179

-; v. Wounds Muskel, v. Wounds Myocarde, v. Cardiac necroses Myologiø, v. Anatomie

Nebennierenrinde und Elektroschock (I. Das Verhalten der doppelbrechenden

Substanzen der Nebennierenrinde bei experimentell ausgelöstem

epileptischem Anfall) 353

Nekrologos vor 1

Nerve terminations in the intervertebral discs of man, ontogenetic develop

ment (Histology of intervertebral discs, 11th communication) .... 96

Nerven, periphere, postmortale Veränderungen $\quad 34$

Nerven (Nervensystem, Zentralnervensystem), v. Anatomie, Catechol amines, Cerebellum, Cerebrum, Chlorpromazine, Cisterna ponto-cere-bellaris, Ganglion ovarien, Hypothalamus, Innervation, Malformations, Nerve terminations, Nerves, Nervous elements, Neuroanatomy, Neurons, Neurosekretion, Sehrinde, Topométrie

Nerves, adrenergic, demonstration with the osmic acid-sodium iodide technique $\quad 379$

Nervous elements, intrinsic, of the digestive tract under experimental con ditions, contribution to the study of their degenerative and regenera

tive changes $(\mathrm{C}) \quad 163$

Neuroanatomy, human (Strong and Elwyri) (4th ed.) (B) 186

Neurochirurgie, v. Topométrie

Neurohistologie, v. Postmortale Veränderungen

Neurohypophysis, v. Neurosekretion

Neurologie, praktische, v. Topométrie

Neurons; postnatal changes in volumetric and density relationships of neurons in cerebral cortex of cat 291

Neuroplegicum, v. Chlorpromazine

Neurosekretion; Anatomie des Hypothalamus des Bibers (castor canadensis),

nebst morphologischen Aspekten der Neurosekretion 126

Niere; Gefäßarchitektur der Niere. Untersuchungen an der Hundeniere

(Heft 5 der «Zwanglosen Abhandlungen aus dem Gebiet der normalen

und pathologischen Anatomie »)(B) 387

-; v. Ureteranomalien

Nodi lymphatici iliaci (lumbales, sacrales etc.), v. Lymphknoten

Nomenclature, v. Nomina anatomica

Nomina anatomica; les nouveaux termes anatomiques (Lexicon conforme à

la nomenclature Internationale PNA) (B) 184

Noradrenaline, v. Adrenergic nerves, Catechol amines

Noyau, v. Nucleus, Zellkern

Nuclear-cytoplasmic ratio, v. Neurons

5-Nucleotidase and alkaline phosphatase activity in septic and aseptic

wounds of rats 270

Acta anat.. Vol. 38, No. 4 (1959) 
400

Register rerum

Nucleus; die morphologische Erfassung der Eiweißstrukturen des Zellkerns

mittels Anthozyan-Rosolsäure (C) 166

-, v. Sex-Chromatin

Nucleus caudatus, v. Catechol amines

Nucleus supraopticus; contribution à $\Gamma$ étude de $\Gamma$ effet de la chlorpromazine

sur le noyau supraoptique (C) 167

-; v. Hypothalamus

02 , v. Tératogénèse

Oberkieferanlage, rudimentäre, v. Schädelentwicklung

Obésité, v. Age changes

Occipitale, ligne courbe occipitale; morphologie de la ligne courbe occipitale

supérieure et ses rapports avec le sinus occipital (C) 173

Oculus, v. Malformations, Metachromasia

Odontology, v. Dental infections, Dentes

Oeil, v. Malformations, Metachromasia

Ohr, v. Bogengang

Ontogenesis; on the role of the « apical ridge $>>$ in the development of the chick

embryo limb bud 280

-; Blastogenese des Menschen (B) 288

-; neue Befunde zur Entwicklung der Bauchspeicheldrüse 114

- ; development of the deciduous cheek teeth of the cat

187

-; v. Malformations, Ontogenetic development, Placenta, Polykaryozyten,

Radicula, Schädelentwicklung, Sehrinde, Tératogénèse, Ureterano-

malien Ontogenetic development of nerve terminations in the intervertebral discs of

man (Histology of intervertebral discs, 11th communication) _.96

Operationen, v. Surgeons Oreille, v. Bogengang

Organa sensuum, v. Anatomie, Mikroskopische Anatomie Oryctolagus cuniculus, v. Adrenergic

nerves, Catechol amines, Schädelentwicklung Os, v. Knochen, Knochennaht, Polykaryozyten,

Tibia Os maxillaire supérieur, v. Schädelentwicklung Os occipitale, v. Knochennaht

Ospubis;lefacteur osseux dans l'étiologie de la hernie inguinale (C) .... 170 Osmic acid-sodium

iodide technique (Champy), v. Adrenergic nerves Osteoarthritis, v. Spinal joint changes

Osteoklasten, v. Polykaryozyten Osteologia, v. Anatomie Otocephalie, v. Schädelentwicklung

Ovarium; contribution à l'étude du ganglion ovarien de poule au cours du

cycle sexuel (C) 168

-; v. Corpus luteum, Gelbkörper

Oviductal epithelium, v. Tuba uterina

Oxygen deficiency, v. Tératogénèse

Oxygène; recherche sur les relations entre $\Gamma$ effet létal et l'effet tératogène de

la carence en oxygène chez l'embryon de poule $\quad 147$

PS2, v. Phosphorus

Pancreas; neue Befunde zur Entwicklung der Bauchspeicheldrüse 114

Register rerum

401

Pancreas; a demonstration of the specific affinity of eosin to the A-cells 
of the Langerhans' 1 islets (C) 171

Paneth cells of the Tamandua tetradactyla Lin., preliminary observations . . 345

Pankreas, v. Pancreas

Parafollikuläre Zellen, v. Thyreoidea

Pars tuberalis der Rinder-Hypophyse, mikroskopischer Aufbau (C) .... 161

Pathogenesis, v. Cardiac necroses

Pathologie, v. Zahngewebe

Peau, v. Anatomie, Haut, Mikroskopische Anatomie

Pelvis, v. Lymphknoten

Periphere Nerven, postmortale Veränderungen $\quad 34$

Phosphatase, alkaline, and 5-nucleotidase activity in septic and aseptic

wounds of rats 270

Phosphorus, radioactive, localization within the spleen at different times 1

after a single dose 135

Pike-perch, v. Zander

Placenta; die Rolle des Fibrins bei der Bildung der menschlichen Placenta . 323

Plaies musculaires, v. Wounds

Plaster slides, v. Metachromasia

Plâtre, blocs de plâtre, v. Metachromasia

Pneumatisation du cornet moyen (morphologie, topographie, intérêt

pratique) (C) 170

Polarisationsmikroskop; die gesunden und erkrankten Zahngewebe des

Menschenund der Wirbeltiere im Polarisationsmikroskop (B) .... 182 Polarisationsmikroskopie,

v. Histochemie, Nebennierenrinde Polarisationsoptische Untersuchung des Zementes entkalkter, retinierter

Zähne 368

Polykaryozyten in fetalen Blutgefäßen (C) 163

Pons, v. Cerebellum

Postmortale Veränderungen in peripheren Nerven

Postnatal changes in volumetric and density relationships of neurons in

cerebral cortex of cat 291

Poule, v. Ganglion ovarien

Poulet, v. Chick embryo, Malformations, Tératogénèse

Poultry, v. Taubenleber

Praxis der Mikrophotographie (2. Aufi.) (B) 385

Premolares, v. Dentes decidui

Prevention, v. Cardiac necroses

Primordialcranium (Kaninchen), v. Schädelentwicklung

Prosophthalmie, v. Schädelentwicklung

Protein, v. Eiweißstrukturen

Rabbit, v. Adrenergic nerves, Catechol amines, Schädelentwicklung

Radicula endomolaris, development (C) $\quad 170$

Radioactive phosphorus, v. Phosphorus

Radioiodine (J131), v. Thyreoidea

«Randleiste», epitheliale, v. ((Apical ridge»

Rat, v. Catechol amines, Son, Spleen, Thyreoidea, Wounds 
Rate, v. Spleen

Rayons X, v. Röntgenbild

Receptors, encapsulated, v. Intervertebral discs

402

Register rerum

Regenerating muscle, $v$. Wounds

Regeneration, v. Nervous elements

Regulation of cell metabolism, v. Cell metabolism

Rein, v. Niere, Ureteranomalien

Ren, v. Niere, Ureteranomalien

Reserpine, v. Adrenergic nerves

Resorptionen, biologische, in den Röhrenknochen, mikroskopische Unter suchungen (B) 386

Retained human teeth, v. Dentes

Retinierte Zähne, menschliche, v. Zähne

Rind v. Bovís

Röhrenknochen, biologische Resorptionen, mikroskopische Untersuchungen

(B) $\quad 386$

Röntgenbild des normalen Menschen (Atlas und Lehrbuch für Praxis und Klinik)(B) $\quad 179$

Rosolsäure und Anthozyan in der Zytologie (C) 166

-; v. Zellkern

Rücken, v. Surgeons

Sandre, v. Zander

Sauerstoffmangel, v. Tératogénèse

Schädelentwicklung; ein Beitrag zur Schädelentwicklung und zum Symmetrieproblem auf Grund von Untersuchungen am Schädel eines

anchyoten, prosophthalmen, kephalothorakopagen Kaninchens mit

rudimentärer Oberkieferanlage 213

Schall, v. Son

Schizophrenic, v. Sehrinde

Sehnenimpressionen, v. Tibia

Sehrinde, quantitative Untersuchungen (Die individuelle Schwankungsbreite beim Menschen, verbunden mit einigen Bemerkungen über die

Schizophrenie. Die Entwicklung der menschlichen Sehrinde. Die

Volumverhältnisse bei einigen Mammalia) (B) 183

Septic conditions, v. Wounds

Sex, v. Female body, Ganglion ovarien, Gelbkörper, Corpus luteum

Sex-Chromatin-Forschung (C)

Sinnesorgane, v. Anatomie, Mikroskopische Anatomie

Sinus lateral; morphologie de la ligne courbe occipitale superieure et ses

rapports avec le sinus lateral (C)

173

Sinus transversus, v. Sinus lateral

Skelet, v. Anatomie

Skeletal muscle, wounds, v. Wounds

Skeletons, mediaeval, v. Spinal joint changes 
Skin, v. Anatomie, Haut, Mikroskopische Anatomie

Skull, v. Schädelentwicklung

Small intestine, v. Paneth cells

Sociétés, v. Yougoslavie

Society transactions, v. Yougoslavie

Son; contribution à I'étude de Faction du son sur le testicule de la souris (C) 176

Son; contribution à I'étude de Finfluence du son sur la structure de Fhypo-

thalamus du rat (C) 174

Sound, v. Son

Register rerum 403

Souris, v. Mus musculus

Spinal joint changes and dental infections, Supplementum 36 (=1 ad Vol. 38)

Splanchnocranium; Beziehungen des alveolären Profilwinkels zu den vorderen

Trajektoriendes Splanchnocraniums (C) 167

Spleen; the localization of radioactive phosphorus within the spleen at

different times after a single dose 135

Splen, v. Spleen

Spondylarthritis, v. Spinal joint changes

Staining, v. Adrenergic nerves, Anthozyan, Langerhans, islets, Microtechnique Statistische Auswertung, v. Haut, Neurons, Sehrinde, Splanchnocranium Sterility, v. Tuba uterina Steroide, v. Cardiac necroses Stress, v. Cardiac necroses Stress auditif, v. Son Stríx aluco, v. Aqueous humor

Submicroscopical structure, v. Ultrastructure, Zähne, Zahngewebe Substantia ossea, v. Dentes Sulcus calcarinus, v. Sehrinde Superior maxilla, v. Schädelentwicklung

Surgeons; anatomy for surgeons (Vol. 111: The back and limbs) (B) .... 335 Surrénales, v.

Nebennierenrinde Symmetrieproblem, v. Schädelentwicklung Symposium, v. Cell metabolism Systema digestorium, v. Digestive tract

345184172

147

176

166

332169

164

Tamandua tetradactyla Lin., Paneth cells, preliminary observations .... Tamanoir, v. Paneth cells

Taschenbuch, biochemisches (B)

Taubenleber; Beitrag zur Kenntnis der Geflügel-, insbesondere der Tauben-

leber(C)

Tawny owl, v. Aqueous humor

Teeth, v. Dental infections, Dentes, Dentes decidui, Zahngewebe

Tératogénèse; recherche sur les relations entre l'effet létal et $\Gamma$ effet tératogène

de la carence en oxygène chez $\Gamma$ embryon de poule

Teratologie, v. Malformations, Mißbildung Terminaisons nerveuses, v. Intervertebral discs

Terminologie, v. Nomina anatomica

Testicule de la souris, etude de Faction du son (C)

Testis, v. Testicule

Therapy, v. Cardiac necroses, Surgeons 
Thyroïde, glande thyroïde, v. Thyreoidea

Thyreoidea; a contribution to morphology of the thyroid in euthermic rat (C)

-; données histochimiques sur les cellules parafolliculaires de la glande

thyroïde du chien

-; the effect of radioiodine (J181) upon the thyroid of rat (C)

Tibia; über die Furchen am Condylus lateralis tibiae und über die Sehnen-

impression des lateralen Meniscus (C)

Tissu adipeux, v. Age changes

Tooth, v. Dental infections, Dentes, Dentes decidui, Zahngewebe

Topographie der inneren Hirnteile, v. Topométrie

404

Register rerum

Topométrie cranio-encéphalique chez Thomme (B) 185

Trajektorien, vordere, des Splanchnocraniums, Beziehungen zum alveolären

Profil·winkel (C) 167

Tranquillizer, v. Chlorpromazine

Transplantation, v. Wing bud

Transverse sinus, v. Sinus lateral

Tuba uterina; studies on the morphology and histochemistry of the Fallopian

tube epithelium, Supplementum 37 (=2 ad Vol. 38) Tumoren, v. Krebsforschung, Lymphknoten,

Zytodiagnostik Tumoren (Tumorartige Mißbildungen), v. Malformations

Ultrastructure, biological (B) 180

-; Zähne, Zahngewebe

Upper jaw, v. Schädelentwicklung

Ureteranomalien bei Embryonen des Rindes

79

Uterus, v. Hysteroectomies

Vaisseaux, v. Arterial supply, Arterio-venöse Anastomosen, Blutgefäße, Gefäßarchitektur,

Malformations, Sinus lateral, Vascularisation

Vaisseaux (vitellins), v. Malformations

Vascularisation of bladder in connection with haematuria vesicalis (C) ... 160

Vegetative Nerven, v. Adrenergic nerves, Digestive tract

Venen, v. Arterio-venöse Anastomosen, Sinus lateral

Vertebrae, v. Spinal joint changes

Vertebral column, joints, v. Spinal joint changes

Vertebrata, v. Zahngewebe

Vesica urinaria, v. Bladder, Hysteroectomies

Vessels, v. Arterial supply, Arterio-venöse Anastomosen, Blutgefäße, Gefäßarchitektur,

Malformations, Sinus lateral, Vascularisation

Vessels (vitelline vessels), v. Malformations

Vessie, v. Bladder, Hysteroectomies

Visual area, v. Sehrinde

Visual centre, v. Sehrinde

Vitellins, vaisseaux vitellins; malformations encéphaliques et microphtalmie gauche après section des vaisseaux vitellins gauches chez $\Gamma$ embryon de

poulet 1

Vitreous body, v. Metachromasia 
Volaille, v. Taubenleber

Volume des neurones, v. Cortex cerebri

Volumetric and density relationships, v. Cortex cerebri

Vorderkammer (im Auge des Waldkauzes), v. Metachromasia

Waldkauz, v. Aqueous humor

Weiblicher Körper, v. Female body

Wing bud; on the role of the «apical ridge» in the development of the chick

embryo limb bud 280

Wirbelsäule, Gelenke, v. Spinal joint changes

Wirbeltiere, v. Zahngewebe

Women, v. Female body, Lymphknoten

Register rerum 405

Wounds; 5 -nucleotida $1 / 8$ e and alkaline phosphatase activity in septic and aseptic wounds of rats 270

Wunden (des Skeletmuskels), v. Wounds

$\mathrm{X}$-rays, v. Röntgenbild

Yougoslavie; comptes-rendus de la reunion annuelle des anatomistes yougoslaves(1958)(C) $\quad 160$

Zähne; polarisationsoptische Untersuchung des Zementes entkalkter, reti nierter Zähne 368

-; v. Dental infections, Dentes, Zahngewebe

Zahngewebe, gesunde und erkrankte, des Menschen und der Wirbeltiere, im

Polarisationsmikroskop (B) 182

Zahnretention, v. Zähne

Zahnzement, v. Zähne

Zander (Lucíopßrca sandra), Gelbkörper (C) 174

Zelldichte, v. Neurons

Zellkern; die morphologische Erfassung der Eiweißstrukturen des Zellkerns

mittels Anthozyan-Rosolsäure (C) 166

Zellvolumen, v. Neurons

Zement entkalkter, retinierter Zähne, polarisationsoptische Untersuchung 368

- , v. Zahngewebe

Zwischenwirbelscheiben, v. Nerve terminations, Spinal joint changes

Zytodiagnostik, gynäkologische, Atlas und Leitfaden (B) 386

Zytologie; Anthozyan und Rosolsäure in der Zytologie (C) 166

-; gynäkologische (Beiträge zur Krebsforschung Bd. 4) (B) 183

Zyto..., v. Cyto...

Book Reviews - Livres Nouveaux - Buchbesprechungen

Baker, J. R.: Principles of biological microtechnique (A study of fixation

and dyeing) (Methuen \& Co. Ltd., London 1958) 178

Bßnnínghoff, A./K. Goerttler: Lehrbuch der Anatomie des Menschen. 1. Bd.:

Allgemeine Anatomie und Bewegungsapparat (6. Aufl.); 3. Bd.:

Nervensystem, Haut und Sinnesorgane (5. Aufl.) (Urban und Schwar-

zenberg, München/Berlin/Wien 1957)

177

CIBA-Foundation symposium on the regulation of cell metabolism (if. Krebs, Editor) (J. \& A. Churchill Ltd., London 1959) 185 
Clara, M.: Die arterio-venösen Anastomosen. Anatomie, Biologie, Pathologie

(2. Aufl.) (Springer Verlag, Wien 1956) 180

Coërs, C. and A. L. Woolf: The innervation of muscle. A biopsy study

(Blackwell Scientific Pubhcations, Oxford 1959) 179

Danielli, J. F. (Editor): General cytochemical methods Vol. 1 (Academic

Press Inc., New York 1958) 388

Delmas, A. et B. Pertuíset: Topométrie cranio-encéphalique chez l'homme

(Masson et Cie., Paris et Ch. C Thomas Springfield, Illinois, USA 1959) . 185 Engström, A. and

J. B. Finean: Biological ultrastructure (Academic Press,

Inc., New York 1958) 180

406

Register rerum

Graumann, W. und K. Neumann (Editores): Handbuch der Histochemie

Bd. I: Allgemeine Methodik, 1. Teil: Gefriertrocknung, Fluoreszenz-

mikroskopie, Polarisationsmikroskopie, Mikrospektrophotometrie,

Mikroradiographie, Autoradiographie, Immunhistologie (Gustav

Fischer Verlag, Stuttgart 1958) 387

Haug, H.: Quantitative Untersuchungen an der Sehrinde. (Die individuelle

Schwankungsbreite beim Menschen verbunden mit einigen Bemerkun-

gen über die Schizophrenie. Die Entwicklung der menschlichen Seh

rinde. Die Volumverhältnisse bei einigen Mammalia.) (Verlag Georg

Thieme, Stuttgart 1958) 183

Heunert, H.-H.: Praxis der Mikrophotographie (2. Aufl.) (Springer-Verlag, Berlin/Göttingen/Heidelberg1959) 385

Hollinshead, W. H.: Anatomy for surgeons (Vol. 111: The back and limbs)

(Cassel \& Co. Ltd., London 1958) 385

Igel, H.: Gynäkologische Zytodiagnostik. Atlas und Leitfaden. (Verlag

Walter de Gruyter \& Co., Berlin 1959) 386

Kügelgen, A. v., B. Kuhlo, W. Kuhlo und K1.-J. Otto: Die Gefäßarchitektur

der Niere. Untersuchungen an der Hundeniere (Heft 5 der «Zwanglosen

Abhandlungen aus dem Gebiet der normalen und pathologischen

Anatomie». Herausgegeb. v. W. Bargmann und W. Doerr) (Georg

Thieme Verlag, Stuttgart 1959) 387

Maty as, J.: Mikroskopische Untersuchungen der biologischen Resorptionen

in den Ròhrenknochen (Akadémiai Kiadó, Budapest 1955) 386

Mazanec, K.: Blastogenese des Menschen (Verlag G. Fischer, Jena 1959) . . 288

Meschan, I., R.M.F. Farrer-Meschan und H. Peisker: Das Röntgenbild des

normalen Menschen (Atlas und Lehrbuch für Praxis und Klinik)

(Medica Verlag, Stuttgart/Wien/Zürich 1958) 179

Möllendorff, W. v./W. Bargmann (Editores): Handbuch der mikroskopi-schen Anatomie des

Menschen Bd. 3: Haut und Sinnesorgane, 3. Teil: E. Horstmann: Die Haut; A. Dabelow: Die

Milchdrüse (Ergänzung zu Bd. HI/1) (Springer-Verlag, Berlin/Göttingen/Heidelberg 1957) . .

181,182

Olivier, G.: Les nouveaux termes anatomiques (Lexique conforme à la

nomenclature Internationale (PNA)). (Vigot Frères, Editeurs, Paris

1959) 184 
Rauen, H. M. (Editor): Biochemisches Taschenbuch (Springer-Verlag, Berlin/Göttingen/Heidelberg 1956) 184

Selye, H.: The chemical prevention of cardiac necroses (The Ronald Press Comp., New York 1958) 289

Schmidt. W. J. und A. Keíl: Die gesunden und die erkrankten Zahngewebe des Menschen und der Wirbeltiere im Polarisationsmikroskop. (Verlag C. Hanser, München 1958) 182

Schroder, R. (Editor): Beiträge zur Krebsforschung Bd. 4: H. Runge (Edi-. tor): Gynäkologische Zytologie (Verlag Th. Steinkopff, Dresden/

Leipzig 1954) 183

Truex, R. C.: Strong and Elwyn's human neuroanatomy (4th ed.) (The

Williams and Wilkins Co., Baltimore 1959) 186

Wallraff, J.: Leitfaden der Histologie des Menschen (2. Aufl.) (Verlag Urban und Schwarzenberg, München/Berlin 1958) 289 